\title{
Application of the z-transform to composite materials
}

\author{
Yi Qin Gao and R. A. Marcus \\ Noyes Laboratory of Chemical Physics, California Institute of Technology, Pasadena, California 91125
}

(Received 21 May 2001; accepted 17 September 2001)

Applications of the $z$-transform were made earlier to interfacial electron transfer involving semi-infinite solids, e.g., semiconductor/liquid and metal/liquid interfaces and scanning tunneling microscopy. It is shown how the method is readily adapted to treat composite materials, such as solid/solid interfaces or "molecular wire"/solid interfaces. (c) 2001 American Institute of Physics. [DOI: 10.1063/1.1416127]

\section{INTRODUCTION}

The electronic structure of solids having surfaces has been of much interest in a variety of physical and chemical studies. ${ }^{1-4}$ Among the simple theoretical methods used for treating the electronic properties of solids the tight-binding approach is considered the simplest that is also reliable for approximate calculations. ${ }^{1,3}$ In the implementation of this method to solids with surfaces, the solids can be considered as consisting of coupled atomic layers parallel to the surface. The system can then be simplified as a one-dimensional chain, with each unit representing a principal layer. ${ }^{5,6}$ The principal layers are then treated separately using the twodimensional space group symmetry.

Various methods have been developed in tight-binding studies of solids having surfaces. In the "slab" $\operatorname{method}^{7}$ the solid is treated as consisting of a finite number of principal layers parallel to the surface and the electronic structure of such a slab is usually obtained by direct diagonalization of the Hamiltonian. The elements of the Hamiltonian are expressed in terms of atomic or molecular orbitals and their interactions within and between layers. Other methods for semi-infinite solids include the transfer matrix ${ }^{6,8}$ and scattering-theoretic $^{9}$ formalisms, which usually employ Green function techniques.

Instead of the Green function method, a $z$-transform method has also been used to treat the electronic structure of a semi-infinite solid. ${ }^{10}$ The $z$-transform, also known as the discrete Laplace transform, ${ }^{11}$ had been applied earlier in electrical engineering and allied fields. The transform reduces the problem of solving an infinite set of linear difference equations to an algebraic equation. This transform method can be applied to multi-band and/or complex interacting systems and still be transparent in its mathematical results. It was recently used to obtain the electronic wave functions of single element $(\mathrm{Si})$ and compound element (InP) semiconductors. ${ }^{12}$ The calculated electronic wave functions were then used to calculate the electronic coupling matrix element for electron transfer reactions at semiconductor/ liquid interfaces. The $z$-transform method proved to be efficient and the results showed good agreement with those of the slab method in those studies.

The Green function approach lends itself quite naturally to focusing on local regions, ${ }^{13,14}$ such as defects or surfaces. It is adaptable to thermal averaging, and there are welldefined procedures for treating Green functions and summing over formal expansions. The wave function approach has been more commonly used in chemical studies, particularly in the form of a slab or cluster approach, since it has permitted very detailed electronic structure calculations. The Green function has been extensively used in recent years in the treatment of "molecular wires" and related systems. ${ }^{14}$

In earlier studies, the $z$-transform was applied to the treatment of semi-infinite solids, ${ }^{10}$ including electron transfer reactions for scanning tunneling microscopy, ${ }^{15,16}$ semiconductor/liquid $^{12}$ and metal/attached monolayer systems. ${ }^{17}$ In the present paper the method is extended to composite systems, such as solid/solid interfaces. The extension also applies to "molecular wire"/solid interfaces, as a particular example of a composite system. Interfaces between a metal and a semiconductor and that between two semiconductors have been studied extensively in the literature, ${ }^{18-20}$ and reflect the scientific and technological interest in such systems. Their electronic structures have been studied by Green function techniques, using tight-binding ${ }^{3,18}$ or pseudopotential methods. ${ }^{2}$ In the present paper the electronic wave function of such systems is obtained by introducing separate $z$-transforms for the coefficients of both solids and using the interaction parameters between the two solids. Both bulk and interfacial states can be studied in this manner.

\section{THE $z$-TRANSFORM METHOD FOR TREATING SEMI-INFINITE SOLIDS AND SOLID/SOLID INTERFACES}

\section{A. $z$ transform}

We have noted earlier the use of "principal layers," which are parallel to the surface, and the subsequent treatment of the system is one-dimensional, each unit being a principal layer. ${ }^{5}$ In the following, we first consider the tightbinding wave functions for a one-dimensional solid-solid interface, and show how the $z$-transform method can be applied to it. For its application to solid/solid interfaces, it is useful to first illustrate the $z$-transform method by applying it to electronic wave functions of a semi-infinite solid. A more 
detailed derivation is given in Ref. 10. In this approach the tight-binding type Hamiltonian $H$ and electronic wave functions $\Psi_{k}$ can be written as

$$
\begin{aligned}
& H=\alpha_{1}|1\rangle\left\langle 1\left|+\alpha \sum_{n=2}^{\infty}\right| n\right\rangle\langle n|+\left(\beta \sum_{n=1}^{\infty}|n+1\rangle\langle n|+\text { c.c. }\right), \\
& \left|\Psi_{k}\right\rangle=\sum_{n=1}^{\infty} c_{n}(k)|n\rangle,
\end{aligned}
$$

where $\quad \alpha_{1}=\langle 1|H| 1\rangle, \quad \alpha=\langle n|H| n\rangle$ (for $n \geqslant 2$ ), $\quad \beta=\langle n$ $+1|H| n\rangle$ (for $n \geqslant 1$ ), and the coefficient $c_{n}(k)$ in Eq. (2) is the solution of an infinite set of linear equations,

$$
\beta^{*} c_{n+1}+(\alpha-E) c_{n}+\beta c_{n-1}=0, \quad n \geqslant 2,
$$

with the boundary condition

$$
\beta^{*} c_{2}+\left(\alpha_{1}-E\right) c_{1}=0 .
$$

In the notation the $k$-dependence of the $c_{n}$ is suppressed for brevity.

To solve the linear equations given by Eq. (3), the $z$-transform for $c_{n}$ is defined by

$$
\mathcal{Z}\left(c_{n}\right)=\sum_{n=1}^{\infty} c_{n} z^{1-n} \equiv F(z),
$$

which, using Eqs. (3) and (4), can be shown to yield ${ }^{10}$

$$
F(z)=c_{1} \frac{\left(\alpha-\alpha_{1}\right) z+\beta^{*} z^{2}}{\beta^{*} z^{2}+(\alpha-E) z+\beta} .
$$

The coefficients $c_{n}$ are recovered using the inverse $z$ transform of $F(z)$,

$$
c_{n}=\frac{1}{2 \pi i} \oint_{C} F(z) z^{n-2} d z,
$$

that is

$$
c_{n}=\frac{c_{1}}{2 \pi i} \oint_{C \beta^{*} z^{2}+(\alpha-E) z+\beta} z^{n-1} d z .
$$

The latter is readily integrated using Cauchy's residue theorem. The $z$-transform is applied to solid/solid interfaces in the next section. For simplicity, the solid/solid interfaces will be studied using mainly one-dimensional models, but the results are immediately generalized to the 3D case. Ideal interfaces and reconstructed interfaces are treated separately in the following. The constant $c_{1}$ can be evaluated by normalizing the wave function to a delta function. ${ }^{10}$

\section{B. Flux, transmission and reflection coefficients}

In the present study we shall need expressions for the probability flux in the tight-binding approximation and for transmission and reflection coefficients at an interface. They are readily obtained: From the time-dependent Schrödinger equation $i \hbar \partial|\Psi\rangle / \partial t=H|\Psi\rangle$, and a wave function $|\Psi\rangle$ $=\Sigma_{m} c_{m}(t)|n\rangle$ and operating on the left by $\langle n|$ we have

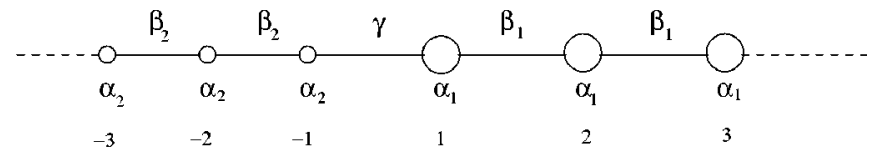

(a)

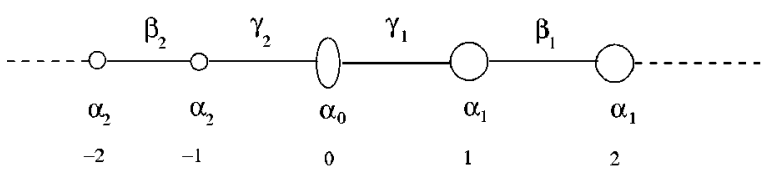

(b)

FIG. 1. Schematic picture of the interface formed by two semi-infinite linear chains: (a) an ideal interface, (b) a reconstructed (coated) interface. $\alpha, \beta$, and $\gamma$ are the interaction parameters. The sites on the left-hand side are denoted by negative numbers and the sites on the right-hand side are denoted by positive numbers.

$$
\begin{aligned}
i \hbar \frac{\partial c_{n}}{\partial t}= & \langle n|H| n+1\rangle c_{n+1}+\langle n|H| n-1\rangle c_{n-1} \\
& +\langle n|H| n\rangle c_{n} .
\end{aligned}
$$

Upon multiplying by $c_{n}^{*}$ and subtracting the complex conjugate we have

$$
\begin{aligned}
\frac{\partial}{\partial t}\left|c_{n}\right|^{2}= & \frac{1}{i \hbar}\left[\left(\langle n-1|H| n\rangle c_{n-1}^{*} c_{n}-\text { c.c. }\right)\right. \\
& \left.-\left(\langle n|H| n+1\rangle c_{n}^{*} c_{n+1}-\text { c.c. }\right)\right] .
\end{aligned}
$$

The right-hand side has the form of the flux $S_{n-1 \rightarrow n}$ from site $n-1$ into site $n$ minus the flux $S_{n \rightarrow n+1}$ out of site $n$ into site $n+1$. We have

$$
S_{n-1 \rightarrow n}=\frac{2}{\hbar} \operatorname{Im}\langle n-1|H| n\rangle c_{n-1}^{*} c_{n},
$$

where Im denotes the imaginary part. In the limit where the $c_{n}(t)$ in Eq. (10) vary as $e^{-i E t / \hbar}$ in Eq. (10) yields the usual tight-binding equation, and the time dependence in Eq. (11) for the flux $S_{n-1 \rightarrow n}$ disappears. Equation (11) can be written in a form reminiscent of the continuum case: ${ }^{21}$

$$
S_{n-1 \rightarrow n}=\frac{2}{\hbar} \operatorname{Im} \beta^{*} c_{n-1}^{*} \Delta c_{n},
$$

where $\Delta c_{n}$ is the first-order difference $c_{n}-c_{n-1}$.

The transmission coefficient $T$ at the interface of two solids is given by the ratio of the transmitted and reflected fluxes

$$
T=S_{\text {trans }} / S_{\text {inc }},
$$

while the reflection coefficient $R$ is the ratio of reflected and incident fluxes, $S_{\text {refl }}$ being negative

$$
R=\left|S_{\text {refl }}\right| / S_{\text {inc }} .
$$

\section{Ideal interface between two one-dimensional one-band systems}

The one-dimensional model of the composite interface is illustrated in Fig. 1(a). The wave function of such a system can be written in terms of localized atomic orbitals 


$$
\left|\Psi_{k}\right\rangle=\sum_{n=-\infty}^{\infty} c_{n}|n\rangle \quad(n \neq 0),
$$

where $|n\rangle$ again denotes the orbital localized at the $n$th site.

The tight-binding Hamiltonian of this system can be written as

$$
\begin{aligned}
H= & \sum_{n=-\infty}^{-1} \alpha_{2}|n\rangle\langle n|+\left(\sum_{n=-\infty}^{-1} \beta_{2}|n\rangle\langle n-1|+\text { c.c. }\right) \\
& +\sum_{n=1}^{\infty} \alpha_{1}|n\rangle\langle n|+\left(\sum_{n=1}^{\infty} \beta_{1}|n+1\rangle\langle n|+\text { c.c. }\right) \\
& +(\gamma|1\rangle\langle-1|+\text { c.c. }),
\end{aligned}
$$

where $\beta_{1}$ and $\beta_{2}$ are the interaction parameters $\langle n$ $+1|H| n\rangle$ between the neighboring sites within each of the two semi-infinite chains, denoted by positive and negative numbers, respectively, $\alpha_{1}$ and $\alpha_{2}$ are the corresponding Coulombic parameters, and $\gamma(=\langle 1|H|-1\rangle)$ is the interaction parameter between the two adjacent sites 1 and -1 of the two chains. As can be seen from the Hamiltonian, it is assumed here for simplicity that the interface consists of only two sites ( -1 and 1$)$, and at first the Coulombic parameter $\alpha$ for each of the two sites at the interface is given the same value as that in each semi-infinite solid. When the interfacial potential parameters $\alpha_{1}$ and $\alpha_{-1}$ are different from their bulk values and/or when the interaction involves more than the nearest neighbors, the same derivation is applicable, but the final formulas are more complicated.

Using the same strategy as that used in the semi-infinite solid case a set of difference equations is obtained for the coefficients $c_{n}$ 's, $n=1,2, \ldots, \infty$ and a set for the other coefficients, $n=-1,-2, \ldots,-\infty$, instead of just one set of equations. These two sets are coupled by the coefficients $c_{-1}$ and $c_{1}$,

$$
\begin{aligned}
& \beta_{1}^{*} c_{n+1}+\left(\alpha_{1}-E\right) c_{n}+\beta_{1} c_{n-1}=0, \\
& n=2,3,4, \ldots \quad(\text { solid } 1), \\
& \beta_{1}^{*} c_{2}+\left(\alpha_{1}-E\right) c_{1}+\gamma c_{-1}=0,
\end{aligned}
$$

and

$$
\begin{gathered}
\beta_{2}^{*} c_{n+1}+\left(\alpha_{2}-E\right) c_{n}+\beta_{2} c_{n-1}=0, \\
n=-2,-3,-4, \ldots \quad(\text { solid } 2), \\
\gamma^{*} c_{1}+\left(\alpha_{2}-E\right) c_{-1}+\beta_{2} c_{-2}=0 .
\end{gathered}
$$

The respective $z$ transforms for solid $1(n \geqslant 1)$ and solid 2 $(n \leqslant-1)$ are

$$
F^{+}(z)=\sum_{n=1}^{\infty} c_{n} z^{1-n}, F^{-}(z)=\sum_{n=-1}^{-\infty} c_{n} z^{1+n} .
$$

With these definitions, $F^{+}(z)$ and $F^{-}(z)$ converge when $|z| \leqslant 1$ and $n \rightarrow \infty$ and $n \rightarrow-\infty$, respectively. Application of the $z$ transform to the two sets of difference equations, Eqs. (17), (19), and (21) yield

$F^{+}(z)=\frac{\beta_{1}^{*} z^{2} c_{1}+\left(\alpha_{1}-E\right) z c_{1}+z \beta_{1}^{*} c_{2}}{\beta_{1}^{*} z^{2}+\left(\alpha_{1}-E\right) z+\beta_{1}} \quad$ (solid 1),
$F^{-}(z)=\frac{\beta_{2} z^{2} c_{-1}+\left(\alpha_{2}-E\right) z c_{-1}+z \beta_{2} c_{-2}}{\beta_{2} z^{2}+\left(\alpha_{2}-E\right) z+\beta_{2}^{*}} \quad($ solid 2).

Equations (18) and (20)-(23) yield

$$
\begin{aligned}
& F^{+}(z)=\frac{c_{1} \beta_{1}^{*} z^{2}-c_{-1} \gamma z}{\beta_{1}^{*} z^{2}+\left(\alpha_{1}-E\right) z+\beta_{1}} \quad(\text { solid } 1), \\
& F^{-}(z)=\frac{c_{-1} \beta_{2} z^{2}-c_{1} \gamma^{*} z}{\beta_{2} z^{2}+\left(\alpha_{2}-E\right) z+\beta_{2}^{*}} \quad(\text { solid } 2) .
\end{aligned}
$$

The coefficients obtained by the inverse $z$ transform are

$$
\begin{aligned}
& c_{n}=\frac{1}{2 \pi i} \oint_{C} F^{+}(z) z^{n-2} d z \quad(\text { solid } 1, \mathrm{n}>0), \\
& c_{n}=\frac{1}{2 \pi i} \oint_{C} F^{-}(z) z^{-n-2} d z \quad(\text { solid } 2, \mathrm{n}<0) .
\end{aligned}
$$

For $n=1$ and $n=-1$, integration of Eqs. (26) and (27) yields $c_{1}=c_{1}$ and $c_{-1}=c_{-1}$, as they should.

To obtain solutions which are propagating to infinity in both sides of the solid, $c_{n}$ and $c_{-n}$ should neither vanish nor become infinite as $n \rightarrow \infty$. (We treat bound interfacial states later.) We thus require that the poles of the integrand in Eqs. (26) and (27) lie on the unit circle, a result which implies that the solution $z=z_{1}$ of

$$
\beta_{1}^{*} z^{2}+\left(\alpha_{1}-E\right) z+\beta_{1}=0
$$

and the solution $z=z_{2}$ of

$$
\beta_{2} z^{2}+\left(\alpha_{2}-E\right) z+\beta_{2}^{*}=0
$$

both lie on the unit circle. In a simple case where $\beta_{1}$ and $\beta_{2}$ are real, the solutions of Eqs. (28) and (29) are both of the type $e^{ \pm i \theta}$, but each typically has a different value of $\theta$. We then have

$$
E-\alpha_{1}=\beta_{1}\left(e^{i \theta}+e^{-i \theta}\right)=2 \beta_{1} \cos \theta_{1}
$$

and

$$
E-\alpha_{2}=2 \beta_{2} \cos \theta_{2},
$$

which also serve to relate $\theta_{2}$ to $\theta_{1} \cdot{ }^{22}$

The $c_{n}$ for $n>0$, as discussed earlier, is given by Eq. (26). (The extension to complex values is readily made.) The poles of the integrand of the right-hand side of Eq. (26) occur at $z=\exp \left(i \theta_{1}\right)$ and $z=\exp \left(-i \theta_{1}\right)$. The former gives a term $\exp \left(i n \theta_{1}\right)$ and the latter $\exp \left(-i n \theta_{1}\right)$. After evaluating the residues, we have

$$
\begin{aligned}
c_{n}= & \frac{\left(c_{1} \beta_{1}-c_{-1} \gamma e^{-i \theta_{1}}\right) e^{i n \theta_{1}}}{2 i \beta_{1} \sin \theta_{1}} \\
& -\frac{\left(c_{1} \beta_{1}-c_{-1} \gamma e^{i \theta_{1}}\right) e^{-i n \theta_{1}}}{2 i \beta_{1} \sin \theta_{1}} \quad(n>0, \text { solid } 1) .
\end{aligned}
$$

Similarly, from Eq. (27), we have 


$$
\begin{aligned}
c_{n}= & \frac{\left(c_{-1} \beta_{2}-c_{1} \gamma e^{-i \theta_{2}}\right) e^{-i n \theta_{2}}}{2 i \beta_{2} \sin \theta_{2}} \\
& -\frac{\left(c_{-1} \beta_{2}-c_{1} \gamma e^{i \theta_{2}}\right) e^{i n \theta_{2}}}{2 i \beta_{2} \sin \theta_{2}} \quad(n<0, \quad \text { solid } 2) .
\end{aligned}
$$

The constants $c_{1}$ and $c_{-1}$ are obtained by a normalization and by satisfying a boundary condition at infinity. For example, if a traveling wave in solid $2, e^{i n \theta_{2}}$, is incident from the left in Fig. 1, and is partially reflected at the interface, and a purely outgoing wave, $e^{i n \theta_{1}}$, occurs in solid 1 then the boundary condition is to set the coefficient of the $e^{-i n \theta_{1}}$ term $(n>0)$ in Eq. (32) equal to zero. Thereby,

$$
c_{1} \beta_{1}=c_{-1} \gamma e^{i \theta_{1}}
$$

and so from Eq. (33), denoting the $c_{n}$ for the transmitted wave in solid 1 by $c_{n}^{\text {trans }}$, we have

$$
c_{n}^{\text {trans }}=c_{1} e^{i(n-1) \theta_{1}} \quad(n>0) .
$$

The $c_{n}$ for the incident wave part of Eq. (33) in solid 2 is denoted by $c_{n}^{\text {inc }}$ and, using Eq. (34), is given by

$c_{n}^{\mathrm{inc}}=-\frac{c_{1}}{2 i \gamma \beta_{2} \sin \theta_{2}}\left(\beta_{1} \beta_{2} e^{-i \theta_{1}}-\gamma^{2} e^{i \theta_{2}}\right) e^{i n \theta_{2}} \quad(n<0)$.

The $c_{n}$ for the reflected wave in solid 2 at the interface is

$$
c_{n}^{\mathrm{refl}}=\frac{c_{1}}{2 i \gamma \beta_{2} \sin \theta_{2}}\left(\beta_{1} \beta_{2} e^{-i \theta_{1}}-\gamma^{2} e^{-i \theta_{2}}\right) e^{-i n \theta_{2}} \quad(n<0) .
$$

The incident flux, using Eq. (11), is

$$
S_{\text {inc }}=\frac{\left|c_{1}\right|^{2}}{4 \hbar \gamma^{2} \beta_{2} \sin \theta_{2}}\left[\beta_{1}^{2} \beta_{2}^{2}-2 \beta_{1} \beta_{2} \gamma^{2} \cos \left(\theta_{1}+\theta_{2}\right)+\gamma^{4}\right] \text {. }
$$

The reflected flux is

$$
\begin{aligned}
S_{\text {refl }}= & -\frac{\left|c_{1}\right|^{2}}{4 \hbar \gamma^{2} \beta_{2} \sin \theta_{2}}\left[\beta_{1}^{2} \beta_{2}^{2}-2 \beta_{1} \beta_{2} \gamma^{2}\right. \\
& \left.\times \cos \left(\theta_{2}-\theta_{1}\right)+\gamma^{4}\right]
\end{aligned}
$$

and the transmitted flux is

$$
S_{\text {trans }}=\frac{\left|c_{1}\right|^{2} \beta_{1} \sin \theta_{1}}{\hbar} .
$$

It is readily verified that $S_{\text {inc }}+S_{\text {refl }}=S_{\text {trans }}, S_{\text {refl }}$ being negative. The ratios $T$ and $R$ in Eqs. (13) and (14) are immediately obtained from Eqs. (38)-(40).

Two limiting cases are readily retrieved from Eqs. (32)(34): In the limiting case where $\gamma=0$, the two semi-infinite solids are uncoupled, and the above expressions yield wave functions which are those of semi-infinite chains. ${ }^{1,10}$ The other limiting case is where the two semi-infinite chains are the same, so that $\gamma=\beta_{1}=\beta_{2}, \alpha_{1}=\alpha_{2}$, and $\theta_{1}=\theta_{2}=\theta$. We then have an infinite one-dimensional chain of sites, and $c_{n}$ $=e^{i n \theta} c_{1}$ for all $n$.
At both metal-semiconductor ${ }^{23}$ and semiconductorsemiconductor interfaces, ${ }^{19}$ bound interfacial states are common and are known to play an important role in determining physical features such as conduction behavior ${ }^{18}$ and the Schottky barrier height. ${ }^{19}$ The bound interfacial states have been studied extensively using the Green function method. ${ }^{24}$ In the following it is seen that the $z$-transform method similarly provides an examination of conditions and energy values for these interfacial states.

To illustrate the use of the $z$-transform method for this purpose, we again model such a situation by a onedimensional chain, again taking the $\beta$ 's and $\gamma$ to be real, for simplicity. In this case, the solutions of Eqs. (28) and (29) are both of the form $z=e^{-\theta_{1,2}}$, where the real parts of $\theta_{1}$ and $\theta_{2}$ are positive. Since $e^{\theta_{1}}$ is also a solution of Eq. (28), in order to avoid $c_{n}$ increasing as $n$ increases, the integrand of Eq. (26) is such that the numerator has $z-e^{\theta_{1}}$ as a factor to cancel a corresponding term in the denominator. Thus we require that

$$
c_{1} \beta_{1} z+c_{-1} \gamma \propto z-e^{\theta_{1}}
$$

which yields

$$
\frac{c_{1}}{c_{-1}}=-\frac{\gamma}{\beta_{1}} e^{-\theta_{1}}
$$

Similarly, we have that

$$
\frac{c_{-1}}{c_{1}}=-\frac{\gamma}{\beta_{2}} e^{-\theta_{2}}
$$

from the requirement that $c_{n}(n<0)$ decreases as $n$ decreases. Comparison of Eqs. (42) and (43) finally yields

$$
e^{\theta_{1}+\theta_{2}}=\frac{\gamma^{2}}{\beta_{1} \beta_{2}} \text {. }
$$

The interfacial states can exist only if Eq. (44) is satisfied, and thus $\gamma^{2} / \beta_{1} \beta_{2}$ must exceed unity. These results are readily extended to three-dimensional cases and complexvalued $\beta$ 's and $\gamma$. The $z$-transform method proves to be a simple method for obtaining the existing condition of interfacial states.

\section{One-dimensional one-band systems with a reconstructed and/or coated interface}

When two solids form a heterojunction, the interface is frequently reconstructed. In many cases one of the solids is coated by some other material beforehand. Such systems have usually been treated by the Green function technique. ${ }^{20}$ This situation is readily modeled by treating the interface as a different unit from the two bulk phases, as illustrated in Fig. 1(b). One type of atom occupies sites from 1 to $\infty$, those of the other type occupy -1 to $-\infty$, and the interface occupies site 0 . Using the same parameters $\alpha_{1}, \alpha_{2}, \beta_{1}$, and $\beta_{2}$ tacitly defined in Eq. (16) and introducing

$$
\alpha_{0}=\langle 0|H| 0\rangle, \quad \gamma_{1}=\langle 1|H| 0\rangle, \quad \gamma_{2}=\langle 0|H|-1\rangle,
$$

the following linear equations are obtained for this system by the same method as that described earlier, 


$$
\begin{aligned}
& \beta_{1}^{*} c_{n+1}+\left(\alpha_{1}-E\right) c_{n}+\beta_{1} c_{n-1}=0, \quad n=2,3,4, \ldots, \\
& \beta_{1}^{*} c_{2}+\left(\alpha_{1}-E\right) c_{1}+\gamma_{1} c_{0}=0,
\end{aligned}
$$

and

$$
\begin{aligned}
& \beta_{2}^{*} c_{n+1}+\left(\alpha_{2}-E\right) c_{n}+\beta_{2} c_{n-1}=0, \quad n=-2,-3, \\
& \quad-4, \ldots, \\
& \gamma_{2}^{*} c_{0}+\left(\alpha_{2}-E\right) c_{-1}+\beta_{2} c_{-2}=0 .
\end{aligned}
$$

Electronic wave functions of such a system can again be obtained using the $z$-transform method, again treating the $\beta$ 's and $\gamma$ as real, for simplicity. Using the boundary condition,

$$
c_{0}=\frac{c_{1} \gamma_{1}+c_{-1} \gamma_{2}}{E-\alpha_{0}},
$$

the tight-binding coefficients are obtained as

$c_{n}=\frac{1}{2 \pi i} \oint_{C} \frac{\left\{c_{1}\left(\beta_{1} z-\frac{\gamma_{1}^{2}}{E-\alpha_{0}}\right)-c_{-1} \frac{\gamma_{1} \gamma_{2}}{E-\alpha_{0}}\right\} z^{n-1}}{\beta_{1} z^{2}+\left(\alpha_{1}-E\right) z+\beta_{1}} d z$,

$c_{n}=\frac{1}{2 \pi i} \oint_{C} \frac{\left\{c_{-1}\left(\beta_{2} z-\frac{\gamma_{2}^{2}}{E-\alpha_{0}}\right)-c_{1} \frac{\gamma_{1} \gamma_{2}}{E-\alpha_{0}}\right\} z^{-(n+1)}}{\beta_{2} z^{2}+\left(\alpha_{2}-E\right) z+\beta_{2}} d z$.

The condition for the existence of bound interfacial states can be found in a way similar to that discussed in the preceding section. For bound interfacial states there should only be terms of exponentially decaying waves in the expression for $c_{n}$. Accordingly, terms that increase as $n$ increases are made to vanish. As in the preceding section, one obtains

$$
\begin{aligned}
& c_{1}\left(\frac{\gamma_{1}^{2}}{E-\alpha_{0}}-\beta_{1} e^{\theta_{1}}\right)=c_{-1} \frac{\gamma_{1} \gamma_{2}}{\alpha_{0}-E}, \\
& c_{-1}\left(\frac{\gamma_{2}^{2}}{E-\alpha_{0}}-\beta_{2} e^{\theta_{2}}\right)=c_{1} \frac{\gamma_{1} \gamma_{2}}{\alpha_{0}-E} .
\end{aligned}
$$

In order for there to be a nontrivial solution for $c_{1}$ and $c_{-1}$, the determinant of the coefficients in Eqs. (53) and (54) vanishes, yielding

$$
\frac{\gamma_{1}^{2}}{\beta_{1}} e^{-\theta_{1}}+\frac{\gamma_{2}^{2}}{\beta_{2}} e^{-\theta_{2}}=E-\alpha_{0} .
$$

The requirement that the wave functions decay in both sides of the solid yields the following condition for the existence of bound interfacial states, obtained by requiring that the poles in Eqs. (51) and (52) be of the form $z=e^{\theta_{1}}$ and $e^{\theta_{2}}$, respectively,

$E=\alpha_{1}+2 \beta_{1} \cosh \theta_{1}=\alpha_{2}+2 \beta_{2} \cosh \theta_{2}, \theta_{1}>0, \quad \theta_{2}>0$.

It is readily verified that the expected results can be obtained for several limiting situations.

A simple semi-infinite system is achieved by setting

$$
\gamma_{1}=\beta_{1}, \quad \gamma_{2}=\beta_{2}=0, \quad \alpha_{2}=0 \text {. }
$$

The condition for the existence of bound surface states can be obtained by applying the above equalities to Eqs. (55) and (56),

$$
\alpha_{1}-\alpha_{0}=\beta_{1} e^{\theta_{1}}, \quad \theta_{1}>0
$$

which can be rewritten as

$$
\frac{\alpha_{1}-\alpha_{0}}{\beta_{1}}>1,
$$

and is in agreement with the known result. ${ }^{1,10}$

Another limiting case occurs when two identical semiinfinite linear chains form an interface

$$
\beta_{1}=\beta_{2}=\gamma_{1}=\gamma_{2}, \quad \alpha_{1}=\alpha_{2} .
$$

It then readily follows that the condition for the existence of bound interfacial states is

$$
\alpha_{1}-\alpha_{0}>0 \text {, }
$$

i.e., that the surface states below the allowed band of the infinite chain can exist only if the $\alpha_{0}$ is more negative than $\alpha_{1}$.

\section{CONCLUDING REMARKS}

In the present study the $z$-transform method has been applied to composite materials, such as solid/solid interfaces. The existing condition for bound interfacial states is obtained in terms of tight-binding solid state parameters, treating both reconstructed and ideally nonreconstructed interfaces. The $z$ transform and the models introduced in the present paper can be applied to systems of experimental interest, including charge transfer through diodes and molecular wires. In particular, it can be applied, using the tight-binding wave functions, to study the interface between a "molecular wire" and a metallic surface ${ }^{25}$ and the contact between nanotubes and metals. $^{26}$

\section{ACKNOWLEDGMENTS}

It is a pleasure to acknowledge the support of this research by the National Science Foundation and the Office of Naval Research.

${ }^{1}$ S. G. Davison and M. Steslicka, Basic Theory of Surface States (Clarendon, Oxford, 1996)

${ }^{2}$ F. Garcia-Moliner and V. R. Velasco, Theory of Single and Multiple Interfaces (World Scientific, Singapore, 1992).

${ }^{3}$ F. Bechstedt and R. Enderlein, Semiconductor Surfaces and Interfaces (Akademie-Verlag, Berlin, 1988).

${ }^{4}$ M. Lannoo and P. Friedel, Atomic and Electronic Structure of Surfaces (Springer-Verlag, Berlin, Heidelberg, 1991).

${ }^{5}$ For example, Ref. 3, pp. 131-133.

${ }^{6}$ D. H. Lee and D. Joannopoulos, Phys. Rev. B 23, 4988 (1981).

${ }^{7}$ For example, K. Hirabayashi, J. Phys. Soc. Jpn. 27, 1475 (1969); C. Calandra, F. Manghi, and C. M. Bertoni, J. Phys. C 10, 1911 (1977); D. J. Chadi, Phys. Rev. B 18, 1800 (1978); K. C. Pandey and J. C. Philips, Phys. Rev. Lett. 34, 1450 (1975).

${ }^{8}$ E. J. Mele and J. D. Joannopoulos, Phys. Rev. B 17, 1816 (1978).

${ }^{9}$ For example, J. Koutecky and M. Tomasek, Phys. Rev. 120, 1212 (1960); I. Ivanov and J. Pollmann, Solid State Commun. 36, 361 (1980). A scattering formalism for STM and related "molecular wire" problems has been used in M. Magoga and C. Joachim, Phys. Rev. B 56, 4722 (1997) and in references to P. Sautet and C. Joachim cited therein; also E. G. Emberly and G. Kirczenow, ibid. 58, 10911 (1998).

${ }^{10}$ R. A. Marcus, J. Chem. Phys. 98, 5604 (1993). 
${ }^{11}$ For example, J. R. Ragazzi and L. A. Zadeh, Trans. Am. Inst. Electr. Eng. 71, 225 (1952); E. I. Jury, ibid. 73, 332 (1954).

${ }^{12}$ Y. Q. Gao, Y. Georgievskii, and R. A. Marcus, J. Chem. Phys. 112, 3358 (2000).

${ }^{13}$ Extensive references to the use of Green function methods are given in Ref. 1, Chap. 7, as well as in J. E. Inglesfield, Prog. Surf. Sci. 25, 57 (1978) and in J. Pollman and S. T. Pantelides, Phys. Rev. B 18, 5524 (1978), papers which describe advantages of a Green function approach.

${ }^{14}$ For example, S. N. Yaliraki, M. Kemp, and M. A. Ratner, J. Am. Chem. Soc. 121, 3428 (1999) and references cited therein; S. Datta, W. Tian, S. Hong, R. Reifenberger, J. I. Henderson, and C. P. Kubiak, Phys. Rev. Lett. 79, 2530 (1997) and references cited therein; C. P. Hsu and R. A. Marcus, J. Chem. Phys. 106, 584 (1997); L. E. Hall, J. F. Reimers, N. S. Hush, and K. Silverbrook, ibid. 112, 1510 (2000).

${ }^{15}$ H. Ouyang and R. A. Marcus, J. Chem. Phys. 100, 7814 (1994).

${ }^{16}$ STM has been treated as a solid-molecule-solid system using a tightbinding model, C. Joachim and P. Sautet, in STM and Related Methods, R. J. Behm, N. Garcia, and H. Rohrer, NATO ASI ser., Ser. E 184, 377 (1990).

${ }^{17}$ S. Gosavi and R. A. Marcus, J. Phys. Chem. B 104, 2067 (2000).

${ }^{18}$ For example, S. G. Davison and J. D. Levine, Solid State Phys. 25, 1 (1970); D. J. Chadi and M. L. Cohen, Phys. Status Solidi B 68, 405 (1975); Y. R. Yang and C. B. Duke, Phys. Rev. B 36, 2763 (1987).
${ }^{19}$ For example, W. Schottky, Z. Phys. 113, 367 (1939); A. Zur, T. C. McGill, and D. L. Smith, Phys. Rev. B 28, 2060 (1983); E. J. Mele and J. D. Joannopolous, ibid. 17, 1528 (1978); J. Tersoff, Phys. Rev. Lett. 52, 465 (1984); Phys. Rev. B 30, 4874 (1984).

${ }^{20}$ For example, W. Moench, J. Vac. Sci. Technol. B 14, 2985 (1996); A. K. Agrawal, Phys. Rev. B 23, 2995 (1981); D. N. Lowy and A. Madhukar ibid. 17, 3832 (1978).

${ }^{21}$ In the continuum case we have instead $S=(\hbar / \mu) \operatorname{Im} \Psi * \partial \Psi / \partial x$. If $a$ denotes the length of a unit cell, and if $\Psi$ is locally normalized to a cell of length $a$, the counterpart of the $\beta$ in Eq. (12) is $\hbar^{2} / 2 \mu a$.

${ }^{22}$ When $\beta_{1}$ and $\beta_{2}$ are not real, Eqs. (32) and (33) again follow, but with the $\beta$ 's replaced by $|\beta|$ 's and the $\theta$ 's each having an additional phase angle.

${ }^{23}$ V. Heine, Phys. Rev. A138, 1689 (1965).

${ }^{24}$ For example, N. V. Dandekar, A. Madhukar, and D. N. Lowry, Phys. Rev. B 21, 5687 (1980); B. Djafari-Rouhani, L. Dobraynski, and M. Lannoo, Surf. Sci. 78, 24 (1978); R. N. Nucho and A. Madhukar, J. Vac. Sci. Technol. 15, 1530 (1978).

${ }^{25}$ S. N. Yaliraki, A. E. Roitberg, C. Gonzalez, V. Mujica, and M. A. Ratner, J. Chem. Phys. 111, 6997 (1999); S. N. Yaliraki and M. A. Ratner, ibid. 109, 5036 (1998).

${ }^{26}$ M. P. Anantram, S. Datta, and Y. Xue, Phys. Rev. B 61, 14219 (2000). 